Analysis Method

\title{
Porosity distribution in laser-sintered polymeric thin sheets as revealed by $\mathrm{X}$-ray micro tomography
}

\author{
A. Liebrich ${ }^{\mathrm{a}, \mathrm{c}, \mathrm{d}, *}$, H.C. Langowski ${ }^{\mathrm{a}, \mathrm{c}, \mathrm{d}}$, R. Schreiber ${ }^{\mathrm{b}, \mathrm{d}}$, B.R. Pinzer ${ }^{\mathrm{b}, \mathrm{e}, \mathrm{f}}$ \\ ${ }^{a}$ Technical University of Munich, Chair of Food Packaging Technology, 85350 Freising, Germany \\ ${ }^{\mathrm{b}}$ University of Applied Sciences Kempten, 87435 Kempten, Germany \\ ${ }^{\mathrm{c}}$ Fraunhofer Institute of Process Engineering \& Packaging IVV, 85354 Freising, Germany \\ ${ }^{\mathrm{d}}$ Kompetenzzentrum für angewandte Forschung in der Lebensmittel- und Verpackungstechnologie (KLEVERTEC), 87437 Kempten, Germany \\ ${ }^{\mathrm{e}}$ Laboratory for Optical 3D Metrology and Computer Vision, University of Applied Sciences Kempten, 87435 Kempten, Germany \\ ${ }^{\mathrm{f}}$ WSL Institute for Snow and Avalanche Research SLF, 7260 Davos Dorf, Switzerland
}

\section{A R T I C L E I N F O}

\section{Keywords:}

Laser Sintering

X-ray micro tomography

Porosity

Porosity distribution

Thin sheets

Thin-walled structures

\begin{abstract}
A B S T R A C T
Laser Sintering (LS) is an additive manufacturing technology that is progressively used to manufacture functional products for end-use applications. However, LS technology provides limited ability to produce thin-walled structures that feature high material quality. A key parameter determining the quality of LS polymeric parts is porosity. This article presents a new approach towards clarifying the porosity characteristics of thin-walled structures produced by LS. Therefore, thin sheets of different thickness built in different orientations within the building chamber are examined by means of X-ray micro tomography. The porosity analysis includes the overall porosity, pore number density, pore size distribution and porosity distribution along the thickness of the thin sheets. The results show that the porosity characteristics strongly depend on the thickness and the build orientation of the thin sheets. This investigation helps to estimate the quality of three dimensional hollow bodies produced by LS and moreover, contributes to a better understanding of the pore formation in LS processes.
\end{abstract}

\section{Introduction}

In recent years, additive manufacturing (AM) has gradually evolved from a prototyping technology to an alternative manufacturing approach besides traditional processing routes. Many industries and fields are investigating the opportunities of AM within their respective domains in order to exploit the unique advantages offered by AM, i.e. increased part complexity, low volume production and even completely customized products. Laser Sintering (LS) is one of the most promising polymer based AM techniques that aims to produce products which feature material properties close to those obtained by common manufacturing technologies, e.g. injection molding [1].

In the LS process a thermoplastic polymer powder is selectively fused layer by layer by means of laser energy. The process consists of three steps that are repeated alternately: At first, a new powder layer is applied to the building platform and preheated to a temperature close to the melting temperature of the polymer by infrared heaters. Secondly, specific areas of the powder layer are melted by a carbon dioxide $\left(\mathrm{CO}_{2}\right)$ laser, while the surrounding powder material remains loosely in the exchangeable frame and forms a supporting structure. In the third step, the building platform is lowered by the thickness of one layer, before another powder layer is applied. Once all parts are completed, the heaters are turned off and cooling takes place. Subsequently, the exchangeable frame is removed from the machine and the loose powder material is detached from the produced parts [2].

A major drawback of LS technology is the limited ability to form thin walls which might be important in particular to create three dimensional hollow bodies. While the resolution along the build direction (z-direction) is determined by the powder layer thickness and the penetration depth of the laser, restrictions parallel to the surface of the powder bed (x-y-direction) mainly depend on the spot size of the laser beam and the scanning strategy applied [3,4]. Seepersad et al. [5] found $0.8 \mathrm{~mm}$ in z-direction and $0.6 \mathrm{~mm}$ in $\mathrm{x}$-y-direction to be the minimal producible thickness for thin walls in LS processes. Design guidelines established by Wegner et al. [4] report on limit values of $0.7 \mathrm{~mm}$ in z-direction and $0.5 \mathrm{~mm}$ in $\mathrm{x}$-y-direction. Moreover, Adam et al. [6] recommend not to produce thin walls of a thickness of less than $1 \mathrm{~mm}$ in any direction in order to prevent high dimensional inaccuracy. Nevertheless, given restrictions may vary depending on the LS machine type, the parameter settings as well as the powder material

\footnotetext{
" Corresponding author. Technical University of Munich, Chair of Food Packaging Technology, 85350 Freising, Germany.

E-mail address: anna.liebrich@ivv.fraunhofer.de (A. Liebrich).
} 
used. Recently, Tasch et al. [7] investigated the mechanical properties of LS thin-walled structures using tensile test samples with thicknesses varying from $0.6 \mathrm{~mm}$ to $2 \mathrm{~mm}$ built in z-direction. The study revealed a significant reduction in mechanical properties for structures with a thickness below $1 \mathrm{~mm}$.

A main parameter critical to the quality of LS parts is porosity. The presence of residual pores within LS polymeric parts is well known and might be attributed to multiple factors, e.g. insufficient consolidation of the powder particles due to low laser energy input or material degradation and vaporization resulting from excessive laser energy input [8]. Until now, the phenomenon of pore formation during LS processes could not have been fully clarified and therefore, a complete control of porosity is still missing. Even for polyamide 12 (PA12), which is the most investigated and applied LS powder material, ensuring low porosity levels remains challenging [9].

Due to its capabilities to non-destructively inspect internal structures, X-ray computed tomography (CT) has been shown to be an effective tool to measure the porosity present within LS parts [10]. Dupin et al. [11] used this technology in order to compare the pore volume distribution of LS parts produced from two different laser sintered PA12 powders and identified the particle size distribution and the crystallization temperature of the powder material as key parameters in the pore formation. Several researcher groups demonstrated a great influence of different LS process parameters on the total pore content and its distribution within LS polymeric parts [12-14]. Apart from that, Pavan et al. [15] revealed that the porosity is significantly affected by the area that is scanned within one layer which determines the time given to the molten polymer to coalesce. Furthermore, Stichel et al. $[16,17]$ showed great differences in the pore morphology between different sample batches produced with different LS machines using different PA12 powders. However, CT based porosity measurements present in literature in general refer to samples cut out of the internal region of large parts, while the borders of the parts are rarely considered. Apart from that, Rüsenberg et al. [18] investigated the porosity at different regions of a LS PA12 cube and found a skin that appears to be significantly denser compared to the internal region.

Until now, only few studies investigated the porosity of small, delicate structures produced by LS. Pavan et al. [19] focused on cylindrical elements with a diameter range typically used in lightweight structure and found the porosity to be significantly influenced by both, the cylinder size and build orientation. Furthermore, Dewulf et al. [20] demonstrated how different laser scanning patterns lead to different radial porosity profiles of small cylinders built in z-direction. However, previous research regarding the porosity of small LS parts in general focused on cylindrical elements, while little work has been done on different geometries, like thin walls. Apart from that, literature mainly reports on samples that were built parallel and vertical to the powder bed platform, whereas little evidence is available on the porosity characteristics of samples built in different orientations. Nevertheless, this might be important in order to estimate the quality of LS three dimensional hollow bodies.

In this study we tested the hypothesis that the porosity within thinwalled structures produced by LS depends on the wall thickness as well as on the orientation in the building chamber. Therefore, disc-shaped thin sheets produced in horizontal, vertical and diagonal orientation with a nominal thickness ranging from $700 \mu \mathrm{m}$ to $2000 \mu \mathrm{m}$ were examined in terms of different porosity characteristics by means of X-ray micro tomography (CT).

\section{Materials \& methods}

\subsection{Production of thin sheets}

Disc-shaped thin sheets with a nominal diameter of $70 \mathrm{~mm}$ and a nominal thickness of $700 \mu \mathrm{m}, 1000 \mu \mathrm{m}, 1500 \mu \mathrm{m}$ and $2000 \mu \mathrm{m}$ were built according to three distinct orientations: parallel to the $x-y$-plane

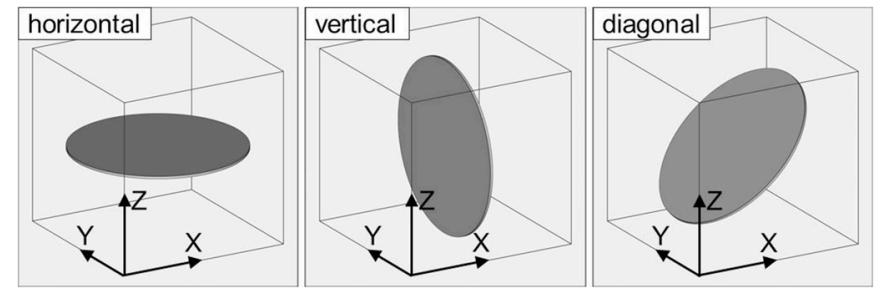

Fig. 1. Orientation of thin sheets within the building chamber (global coordinate system).

(horizontal orientation), in z-direction (vertical orientation) and in a $45^{\circ}$ angle to the $x-y$ plane (diagonal orientation) (see Fig. 1). An overview of the different thin sheets investigated within this work is given in Table 1.

A batch of multiple thin sheets was produced on an EOS (EOS Electro Optical Systems GmbH, Krailling, Germany) P110 LS machine using PA2200 PA12 powder supplied by EOS GmbH with a mixing ratio $50 / 50$ between virgin and recycled powder (particle median size $\mathrm{d}_{50}=56 \mu \mathrm{m}$ ). The process parameters "PA2200 Performance", offered by the LS machine manufacturer EOS GmbH as standard settings for PA2200 PA12 powder at $100 \mu \mathrm{m}$ layer thickness, were applied. The corresponding scanning strategy was an alternating $x-y$ scanning pattern including two contour lines and enclosed raster lines ("hatch lines"). Fig. 2 displays magnified sections of the $x-y$ scanning patterns used to produce the different thin sheets.

Regarding the sheets with a nominal thickness of $700 \mu \mathrm{m}$ built in vertical orientation (sheet $700 \mathrm{~V}$ ), the cross section that is sintered per layer did not provide enough space to create a regular scanning pattern comprising contour lines and hatch lines. Therefore, the system automatically switched from contour to edge exposure which implies that the inner contour was replaced by a single scanning line referring to different exposure parameters (see pink line in the scanning pattern of sheet 700V in Fig. 2).

After the building process, all sheets were finished by bead blasting in order to remove remaining powder material. Per each nominal thickness $(700 \mu \mathrm{m}, 1000 \mu \mathrm{m}, 1500 \mu \mathrm{m}, 2000 \mu \mathrm{m})$ and build orientation (horizontal, vertical, diagonal) considered, one thin sheet was randomly selected for subsequent porosity analysis.

\subsection{X-ray micro tomography}

In order to prepare samples for CT measurements, a rectangular block with dimensions $(25 \times 10) \mathrm{mm}^{2}$ was cut out from the middle of each of the disc-shaped thin sheets investigated. In addition, for all three thin sheets with a nominal thickness of $1000 \mu \mathrm{m}(1000 \mathrm{H}, 1000 \mathrm{~V}$, 1000D) two further equivalent samples were cut out at different positions of the sheets in order to test the homogeneity of porosity within the entire volume of the sheets (see Fig. 3a).

If necessary, the size of the samples had to be slightly adjusted by cutting away some extra material along the long edge in order to loosely fit the sample containers (cylindrical polyetherimide (PEI) tubes with $10 \mathrm{~mm}$ inner diameter) without applying any mechanical distortion. The samples were fixed in the containers by freezing the bottom in $2 \mathrm{~mm}$ deep water (the scanner resided in a cold room at $-20^{\circ} \mathrm{C}$ ).

All samples were scanned using a Scanco $\mu \mathrm{CT} 40$ desktop device with an acceleration voltage of $70 \mathrm{keV}$ and low current setting of $57 \mu \mathrm{A}$ to achieve a small spot size. 1000 projections were recorded per $180^{\circ}$ sample rotation. These settings resulted in a voxel size of $(6 \mu \mathrm{m})^{3}$. Regarding the thin sheet with a nominal thickness of $2000 \mu \mathrm{m}$ built in horizontal orientation $(2000 \mathrm{H})$, the corresponding sample did fail during CT measurement and was therefore not available for further evaluation.

Post-processing of the raw data was equal for all samples and consisted of three steps: 
Table 1

Overview of investigated thin sheets and corresponding samples for X-ray micro tomography (CT) measurements.

\begin{tabular}{|c|c|c|c|c|c|}
\hline thin sheet & build orientation & nominal thickness $[\mu \mathrm{m}]$ & number of CT samples & effective thickness $[\mu \mathrm{m}]$ & volume of interest $(\mathrm{VOI})^{\mathrm{a}}\left[\mathrm{mm}^{3}\right]$ \\
\hline $700 \mathrm{H}$ & horizontal & 700 & 1 & 671.4 & 6.04 \\
\hline $700 \mathrm{~V}$ & vertical & 700 & 1 & 562.4 & 5.06 \\
\hline $700 \mathrm{D}$ & diagonal & 700 & 1 & 647.8 & 5.83 \\
\hline $1000 \mathrm{H}$ & horizontal & 1000 & 3 & $786.0 / 785.9 / 789.1$ & $7.07 / 7.07 / 7.10$ \\
\hline $1000 \mathrm{~V}$ & vertical & 1000 & 3 & $946.0 / 931.5 / 951.0$ & $8.51 / 8.38 / 8.56$ \\
\hline $1000 \mathrm{D}$ & diagonal & 1000 & 3 & $973.1 / 974.0 / 971.5$ & $8.76 / 8.77 / 8.74$ \\
\hline $1500 \mathrm{H}$ & horizontal & 1500 & 1 & 1417.9 & 12.76 \\
\hline $1500 \mathrm{~V}$ & vertical & 1500 & 1 & 1457.4 & 13.12 \\
\hline $1500 \mathrm{D}$ & diagonal & 1500 & 1 & 1543.5 & 13.89 \\
\hline $2000 \mathrm{H}$ & horizontal & 2000 & $\mathrm{n} / \mathrm{a}$ & $\mathrm{n} / \mathrm{a}$ & $\mathrm{n} / \mathrm{a}$ \\
\hline $2000 \mathrm{~V}$ & vertical & 2000 & 1 & 2040.2 & 18.36 \\
\hline $2000 \mathrm{D}$ & diagonal & 2000 & 1 & 2029.0 & 18.26 \\
\hline
\end{tabular}

${ }^{\text {a }}$ For all samples the VOI corresponds to a volume with voxel dimensions of $500 \times 500\left(=9 \mathrm{~mm}^{2}\right)$ times the thickness of the sample.

1 Geometric alignment of all slices of the scanned samples such that the slices were always oriented horizontally in a CT image: This step facilitated subsequent evaluation procedures and was necessary because the placement of the samples in the CT scanner did not allow for a precise control of the rotation angle. The geometric alignment was implemented as an angular offset in the reconstruction algorithm, thus avoiding numerical errors by interpolation that would have been introduced by rotating the slices after reconstruction.

2 Definition of a volume of interest (VOI) and a local coordinate system $(\tilde{x}, \tilde{y}, \tilde{z})$ with the $\tilde{x}$-z-plane parallel to the surface of the corresponding sample: To avoid any boundary effects from cutting, a small cubic subvolume (voxel dimensions of $500 \times 500$ in $\tilde{x}$ - and $\tilde{z}$-direction times the appropriate thickness to completely contain the sample in $\tilde{y}$-direction) was virtually cut out from the entire scanned sample volume for subsequent porosity analysis (see Fig. 3b).

3 Application of a median filter (support 3) and then a Gaussian filter (sigma 1.2, support 3 ) for noise reduction.

All data sets were segmented with an automatically determined threshold according to Otsu [21], resulting in polymeric foreground and air background. The polymeric foreground, being the direct result of segmentation, should consist of only one large component, where all voxels are connected. However, for most of the samples, visual inspection revealed a small number of disconnected voxels close to the interface which might probably result from powder remains. Therefore, the polymeric foreground was subjected to a connected component analysis and only the largest connected component (termed "matrix") was kept for further analysis. To obtain the voxels that belong to the pores, the matrix data set was inverted, resulting in an image where all air voxels are interpreted as objects. Since the samples were surrounded by air during scanning, air voxels could not only be found in the pores enclosed in the matrix, but also surrounding the matrix, i.e. one large air component next to either of the two surfaces. Therefore, the two largest air components were discarded and only the air voxels referring to the pores were kept.

Based on the data for matrix and pores, the following geometrical parameters were calculated:

Effective thickness: Since the LS thin sheets possess quite rough surfaces, CT data was used to measure their effective thicknesses. This parameter was defined as the thickness of an (imaginary) box having the same width (500 voxels in $\tilde{x}$-direction) and height (500 voxels in $\tilde{z}$-direction) as the VOI and containing the same amount of voxels as the matrix and the pores together.

Overall porosity: Overall porosity was determined as the ratio of the number of pore voxels over the number of voxels contained within the total volume comprising pore and matrix volume (number of pore voxels plus number of matrix voxels).

Pore number density: Pore number density was obtained by a)

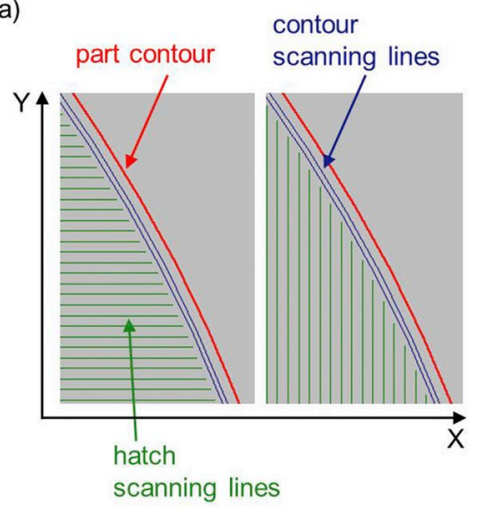

b)

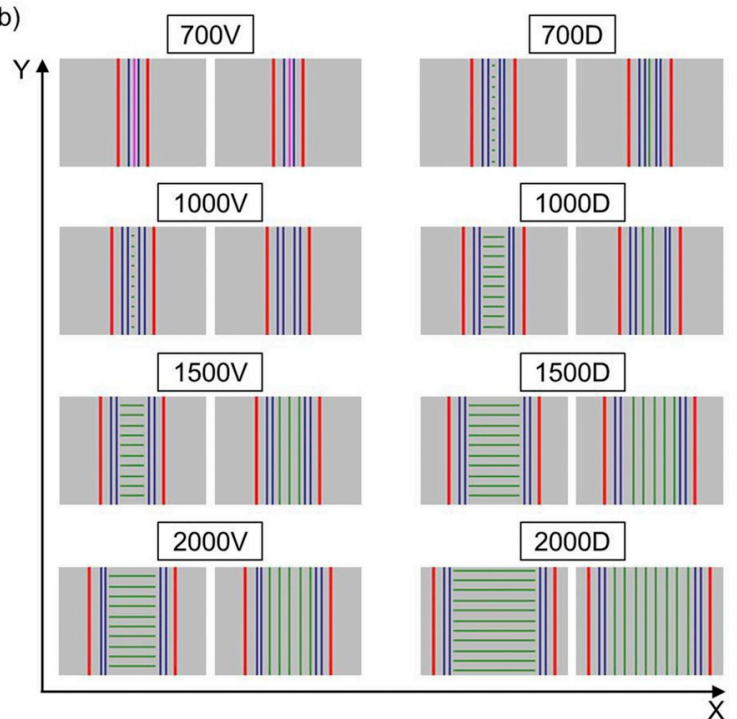

Fig. 2. Magnified sections of $x-y$ scanning patterns used to produce thin sheets in horizontal orientation (a) (the same for all nominal thicknesses investigated) and thin sheets of different nominal thickness in vertical and diagonal orientation (b). 
a)

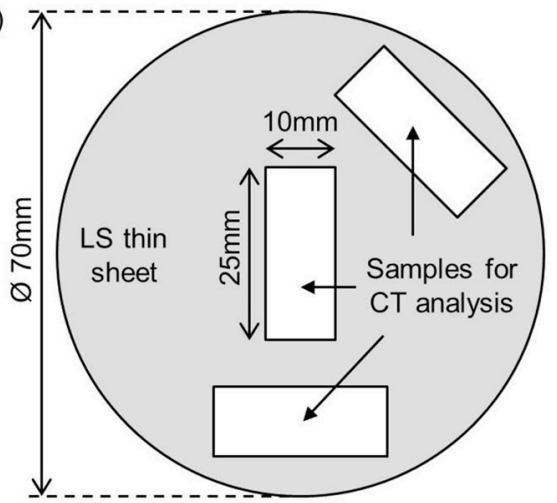

b)

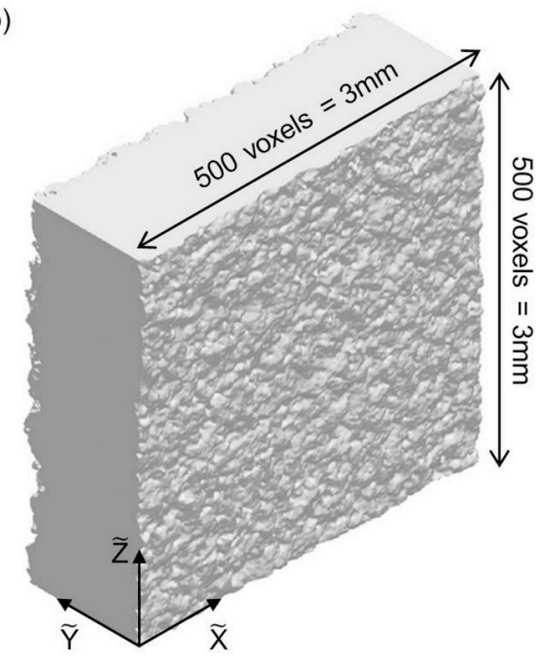

Fig. 3. Sample preparation for X-ray micro tomography (CT) measurements (a) and volume of interest (VOI) with local VOI coordinate system (b).

dividing the number of disjoint pores by the total volume containing pore and matrix volume. Pores of less than 3 voxels (which is a pore volume of $648 \mu \mathrm{m}^{3}$ or an equivalent pore diameter of $10.7 \mu \mathrm{m}$ ) are likely to result from measurement noise, probably leading to erroneous pore detection. The amount of pores smaller than 3 voxels would account for up to $10 \%$ of the total number of pores detected within the thin sheets investigated. To avoid any ambiguity due to resolution limits, only pores consisting of 3 voxels or more were included in the analysis when quantifying the pores by number.

Equivalent pore diameter: For each individual pore, the pore volume was obtained by counting the voxels that define the pore. The equivalent pore diameter was defined as the diameter of an (imaginary) sphere of the same volume.

Two-dimensional (2D) porosity: In order to investigate local porosity inside the thin sheets, a two-dimensional porosity (2D porosity) was defined as the ratio of pore area to slice area for arbitrary slices extracted from the VOI. The slices were aligned parallel to the $\tilde{x}-\tilde{z}$-plane of the local VOI coordinate system which is parallel to the surface of the thin sheets and thus, porosity distribution along the thickness of the thin sheets (in $\tilde{y}$-direction) could be determined.

\section{Results and discussion}

At first, the validity of the CT measurements (3.1.) will be demonstrated, followed by a brief summary of the results for the effective thickness of the different thin sheets investigated (3.2.). Based on this, the porosity analysis of the thin sheets will be presented, in particular focussing on the influence of the thickness and build orientation on different porosity characteristics. This includes the discussion of the overall porosity and pore number density (3.3.1.). Moreover, the pore size distribution (3.3.2.) as well as the porosity distribution within the thin sheets (3.3.3.) will be investigated in detail. Finally, the presence of open pores which are connected to the surface of the thin sheets will be demonstrated (3.3.4.). In order to facilitate the classification of the sheets to different build orientations, the results are presented using a colour scheme with pink referring to horizontal, blue to vertical and orange to diagonal orientation.

\subsection{Validity of CT measurements: representative volume and spatial variability}

Validation of CT measurements has been conducted taking the example of the three thin sheets with a nominal thickness of $1000 \mu \mathrm{m}$ built in horizontal, vertical and diagonal orientation $(1000 \mathrm{H}, 1000 \mathrm{~V}$, 1000D). First of all, it has been tested, whether the defined VOI was representative for the bulk properties of the entire sample volume (rectangular block with dimensions $(25 \times 10) \mathrm{mm}^{2}$ ). Apart from that, three equivalent samples referring to different positions of the sheets were compared in order to investigate the variability of the porosity characteristics within the entire volume of a single sheet.

Fig. 4 presents the calculated overall porosity as a function of the considered data volume for three individual samples prepared from the thin sheets $1000 \mathrm{H}, 1000 \mathrm{~V}$ and 1000D.

Regarding the size of the cubic data volume considered for data evaluation, only the dimensions in $\tilde{x}$ - and $\tilde{z}$-direction (local VOI coordinate system) were varied while the dimensions in $\tilde{y}$-direction were always kept constant to contain the entire thickness of the corresponding sample. From Fig. 4 it is apparent that for all samples a stable value of the overall porosity was reached starting from a data volume of $2 \mathrm{~mm}^{3}$. This allows the conclusion that CT data evaluation based on a VOI with voxel dimensions of $500 \times 500$ in $\tilde{x}$ - and $\tilde{z}$-direction $(=$ $9 \mathrm{~mm}^{2}$ ) times the thickness of the sample in $\tilde{y}$-direction should be representative for the porosity characteristics of the entire sample volume. Comparing overall porosity results for three individual samples referring to different positions in a single thin sheet, the mean values and standard deviations obtained for the sheets $1000 \mathrm{H}, 1000 \mathrm{~V}$ and $1000 \mathrm{D}$ are $1.35 \pm 0.02 \%, 1.78 \pm 0.05 \%$, and $1.43 \pm 0.10 \%$ respectively. This suggests that no significant spatial variations of porosity characteristics occur within the entire volume of one thin sheet.

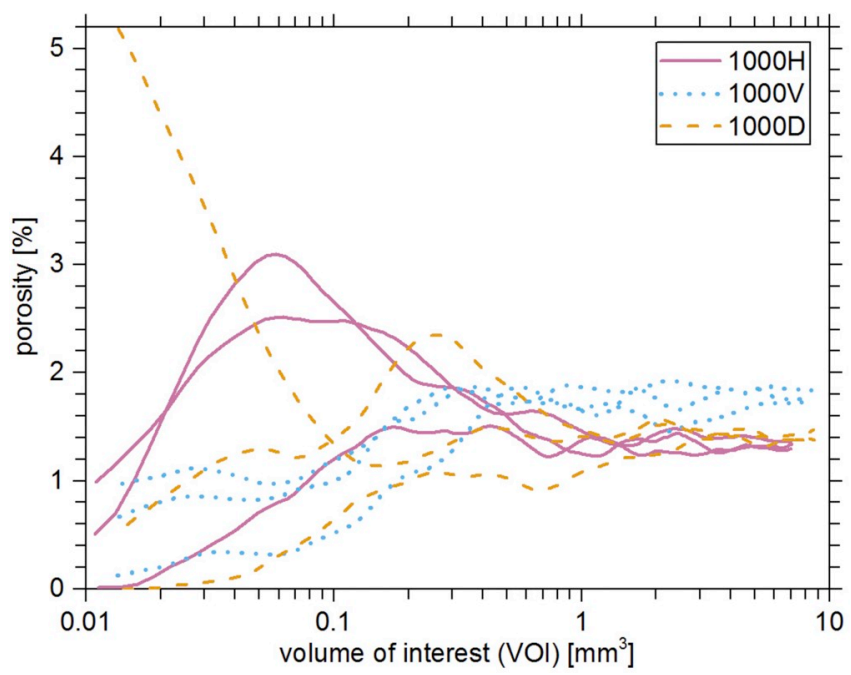

Fig. 4. Overall porosities of three individual samples referring to different positions in the sheets with $1000 \mu \mathrm{m}$ nominal thickness built in horizontal, vertical and diagonal orientation as a function of the volume of interest (VOI). For each sample, VOI $\tilde{y}$-dimensions were kept constant to contain the entire thickness of the sample. 


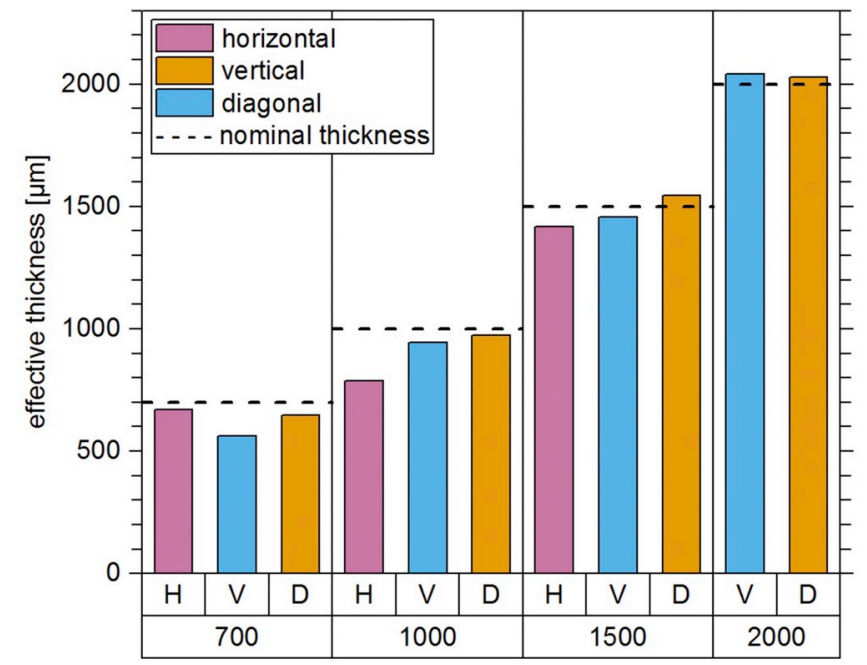

Fig. 5. Effective thicknesses of sheets of different nominal thickness built in horizontal, vertical and diagonal orientation. Values given for sheet $1000 \mathrm{H}$, $1000 \mathrm{~V}$ and 1000D are mean values with standard deviations of $\pm 2 \mu \mathrm{m}$ $(1000 \mathrm{H}), \pm 10 \mu \mathrm{m}$ (1000V) and $\pm 1 \mu \mathrm{m}$ (1000D) calculated from three individual samples referring to different positions in the sheet.

\subsection{Effective thickness}

Fig. 5 depicts the effective thicknesses of the thin sheets with a nominal thickness of $700 \mu \mathrm{m}, 1000 \mu \mathrm{m}, 1500 \mu \mathrm{m}$ and $2000 \mu \mathrm{m}$ built in horizontal, vertical and diagonal orientation.

It can be recognized that the thin sheets mainly show differences between the nominal and effective thickness in the range of $\pm 100 \mu \mathrm{m}$. Apart from that, one observes greater dimensional deviations for the sheets $700 \mathrm{~V}$ and $1000 \mathrm{H}$ that are $-137 \mu \mathrm{m}$ and $-216 \mu \mathrm{m}$ respectively. It is well known that build accuracy of LS technology decreases when the size of the part decreases. In general, the scaling factors applied in order to compensate the shrinkage on cooling are primarily designed for the production of larger parts, leading to low dimensional accuracy when producing small parts [4]. Regarding thin sheets built in vertical and diagonal orientation, resolution limitations might be expected because small nominal thicknesses did not provide enough space to create full scanning patterns comprising both, contour and hatch lines (see Fig. 2b). For sheet $700 \mathrm{~V}$, the scanning strategy with only one contour line and an enclosed edge line produced an effective thickness of $563 \mu \mathrm{m}$ which is significantly smaller compared to the nominal value $(=700 \mu \mathrm{m})$. The great dimensional deviations observed for sheet $1000 \mathrm{H}$ might be traced back to erroneous part design when applying rescaling parameters to the nominal thickness value. This step was conducted in order to compensate the expected oversize in z-direction which is well known for LS thin walls oriented parallel to the x-y-plane and can be explained by the fact, that the laser energy penetrates deeper than through only one layer which, for the first layer, leads to the connection of extra material that should not belong to the part [6]. In order to obtain reliable results, porosity analysis has been conducted comparing different thin sheets according to their effective thickness.

\subsection{Effect of build orientation and effective thickness on porosity}

\subsubsection{Overall porosity and pore density}

Fig. 6a shows the overall porosities of the thin sheets built in horizontal, vertical and diagonal orientation as a function of the effective thickness.

Values are in the range between $0.7 \%$ and $2.6 \%$ and are significantly lower compared to overall porosity levels reported for LS parts of greater dimensions, e.g. tensile bars, which are typically around $3-5 \%[15,17,20]$. Apart from that, there is a progressive increase in the
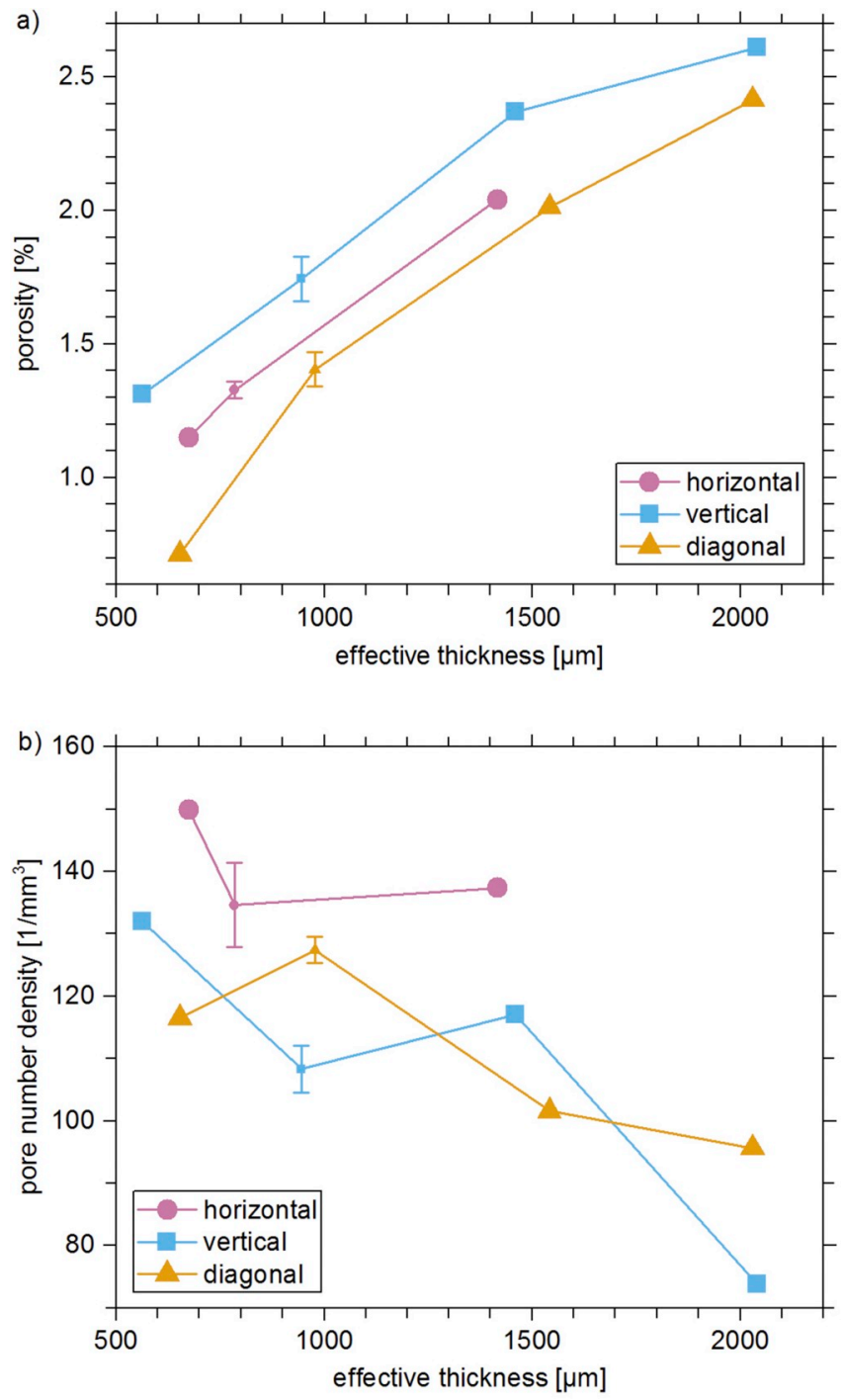

Fig. 6. Overall porosities (a) and pore number densities (b) of detected pores (i.e. $\geq 10.7 \mu \mathrm{m}$ equivalent pore diameter) of sheets built in horizontal, vertical and diagonal orientation as a function of the effective thickness. Error bars present standard deviations calculated from three individual samples referring to different positions in the sheet.

overall porosity level with growing thickness of the sheets for all three build orientations investigated. An explanation can be found when looking at Fig. 7 which displays semi-transparent projections of the visualized CT data sets through $3 \mathrm{~mm}$ width in $\tilde{x}$-direction (local VOI coordinate system) for all thin sheets investigated.

It is apparent that vertically and diagonally built sheets present a pronounced dense skin and a more porous internal region. This effect might primarily result from the contour function of the LS system as indicated by the scanning patterns illustrated in Fig. 2. Compared to the sheets built in vertical and diagonal orientation, horizontally built sheets show pores that are closer to the surface. Nevertheless, also for sheets built in horizontal orientation the local porosity obviously decreases towards both, the upward-facing and downward-facing surface. This is in good agreement with results obtained by Rüsenberg et al. [18], who found a dense skin for LS cubes that were manufactured without contour or edge parameters and formulated the hypothesis, that the thickness of LS parts and the cooling rate influence the outer solid area and the porosity as well. The increase in overall porosity with growing thickness of the thin sheets might primarily result from the 


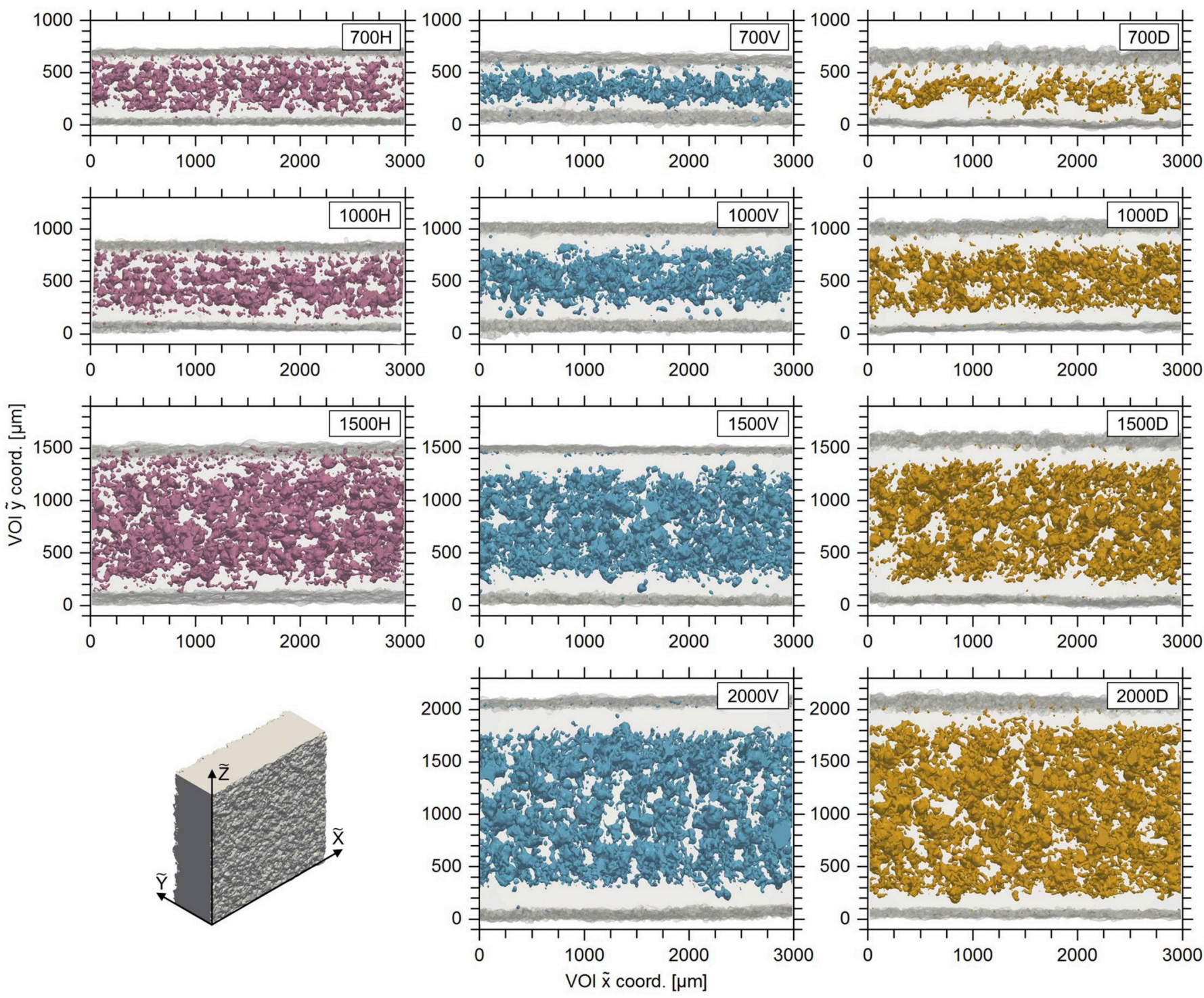

Fig. 7. Semi-transparent projections of visualized CT data sets through $3 \mathrm{~mm}$ ( $=500$ voxels $)$ width in $\tilde{x}$-direction for sheets of different thickness built in horizontal, vertical and diagonal orientation.

different ratio between the more porous internal region and the denser skin. Similar findings were reported by Pavan et al. [19] who investigated the porosity of cylindrical elements with diameters ranging from 0.6 to $6 \mathrm{~mm}$. From a certain cylinder diameter onwards, the contribution of the dense skin to the total volume of the cylinders became negligibly small, leading to a stable overall porosity level which has been considered as equal to the bulk value.

Comparing the graphs referring to different build orientations in Fig. $6 \mathrm{a}$, sheets built in vertical orientation present higher overall porosity values compared to both, horizontally and diagonally built sheets. Pavan et al. [19] found a maximum of porosity located close to the borders of small cylinders built in vertical orientation leading to a higher overall porosity compared to horizontally built cylinders. A similar phenomenon was observed for the vertically built thin sheets investigated within this work as will be discussed in detail in section 3.3.3. Apart from that, lowest overall porosity levels are observed for diagonally built sheets while sheet 700D presents a significant lower value compared to thin sheets of comparable thickness built in different orientations. Imagining the building process in diagonal orientation, the rectangular cross section which is sintered per layer is continuously shifted in $\mathrm{x}$-direction (see Fig. 2b), which means that the sintered area of one layer is only partially overlapped by the sintered area referring to the subsequent layer. This probably might have led to different pore formation and thus could have caused the lower overall porosity results compared to thin sheets built in horizontal and vertical orientation. Since the ratio of non-overlapping area to overlapping area decreases with increasing thickness of the diagonally built sheets, the effect might be more pronounced for small thicknesses and become less visible for thicker sheets.

Fig. $6 \mathrm{~b}$ depicts the number densities of detected pores (i.e. $\geq 10.7 \mu \mathrm{m}$ equivalent pore diameter) for the thin sheets as a function of the effective thickness. Values are ranging between 74 and 150 pores per cubic millimetre, while the pore number density in general tends to decrease when the thickness increases. Taking into account the overall porosity results, which show an increase with growing thickness of the sheets, this might indicate larger pore sizes for thicker sheets. Apart from that, sheets built in horizontal orientation show highest pore counts for all nominal thicknesses investigated, whereas no significant differences can be found comparing vertical and diagonal sheets. The differences in the pore number densities comparing different build orientations moreover are not analogous to the overall porosity behaviour. Thus, one could expect variations in pore size distributions not only depending on the thickness but also on the build orientation of the thin sheets. 

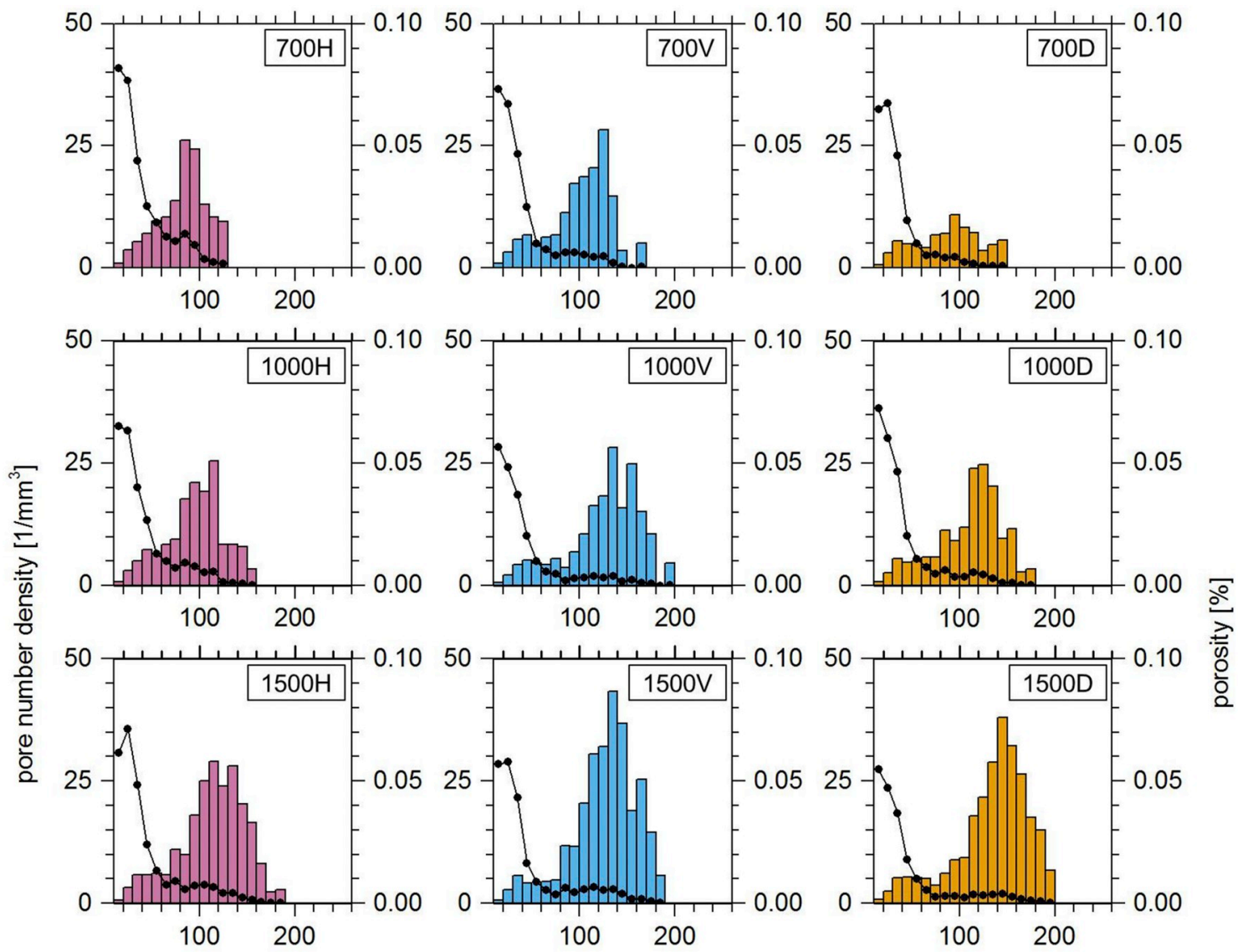

\begin{tabular}{|l|}
\hline- pore number density \\
distribution
\end{tabular}
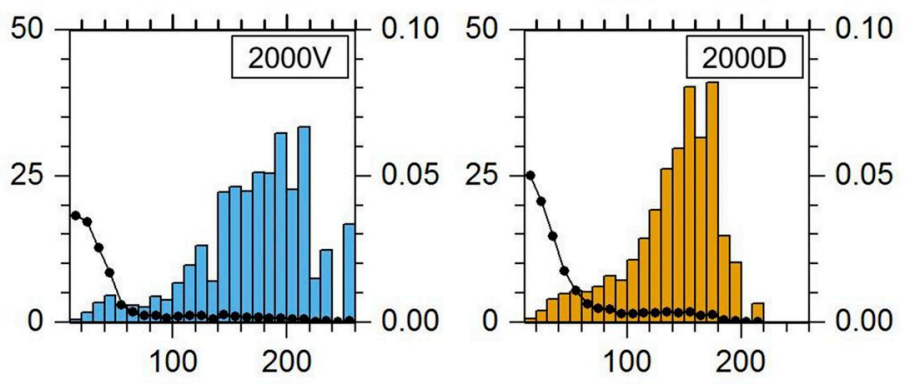

equivalent pore diameter $[\mu \mathrm{m}]$

Fig. 8. Pore size distributions of pore number density and porosity for sheets of different thickness built in horizontal, vertical and diagonal orientation. The equivalent pore diameter is defined as the diameter of an (imaginary) sphere of the same volume as the pore.

\subsubsection{Pore size distribution}

The previous section revealed that the overall porosities and pore number densities measured for the thin sheets with varying thickness built in different orientations are not congruent, indicating that different pore size distributions may exist. More details are now given in Fig. 8, which shows the pore number densities of different pore sizes and their contributions to the overall porosity. In order to inspect the pore morphology, Fig. 9 depicts a semi-transparent projection of the visualized CT data set through the total thickness of the sample in $\tilde{y}$-direction for each of the thin sheets investigated.

From Fig. 8 it is apparent that all sheets exhibit high counts of small pores $(<50 \mu \mathrm{m})$ indicating that pores with sizes close to the resolution limit of the CT analysis (only pores defined by 3 voxels or more which means an equivalent pore diameter equal to or greater than $10.7 \mu \mathrm{m}$ were considered) may also be present in significant amounts. Nevertheless, the total volume of those small pores may be negligibly small compared to larger pore sizes, which allows the conclusion that pores below the CT detection limit would not influence the measured overall porosity levels significantly. By contrast, the pore number densities shown in Fig. 6b would be affected by the presence of smaller pores. The graphs should therefore be interpreted as pore number densities of detected pores.

For all build orientations, pore size distributions appear clearly shifted towards larger pore sizes with increasing thickness. This effect becomes especially evident for thin sheets built in vertical orientation. Regarding sheet 2000V, Fig. 8 reveals pore sizes up to an equivalent pore diameter of $260 \mu \mathrm{m}$. By contrast, significantly smaller pore sizes were found for sheet $700 \mathrm{~V}$, where the largest equivalent pore diameter is $170 \mu \mathrm{m}$. When looking at the visualized data sets displayed in Fig. 9, it becomes evident that larger pore sizes present quite complex shapes resembling a network of interconnected smaller pores. Previous research revealed that reducing scanning speed or increasing laser power yields larger pore sizes with a lower number of small pores, suggesting the progressive coalescence of smaller pores into larger ones with increasing energy density [20,22]. According to Wegner [23], increasing energy density causes a higher absolute temperature level of the polymer melt. Thus, the different pore size distributions comparing thin sheets with different thicknesses could result from different 

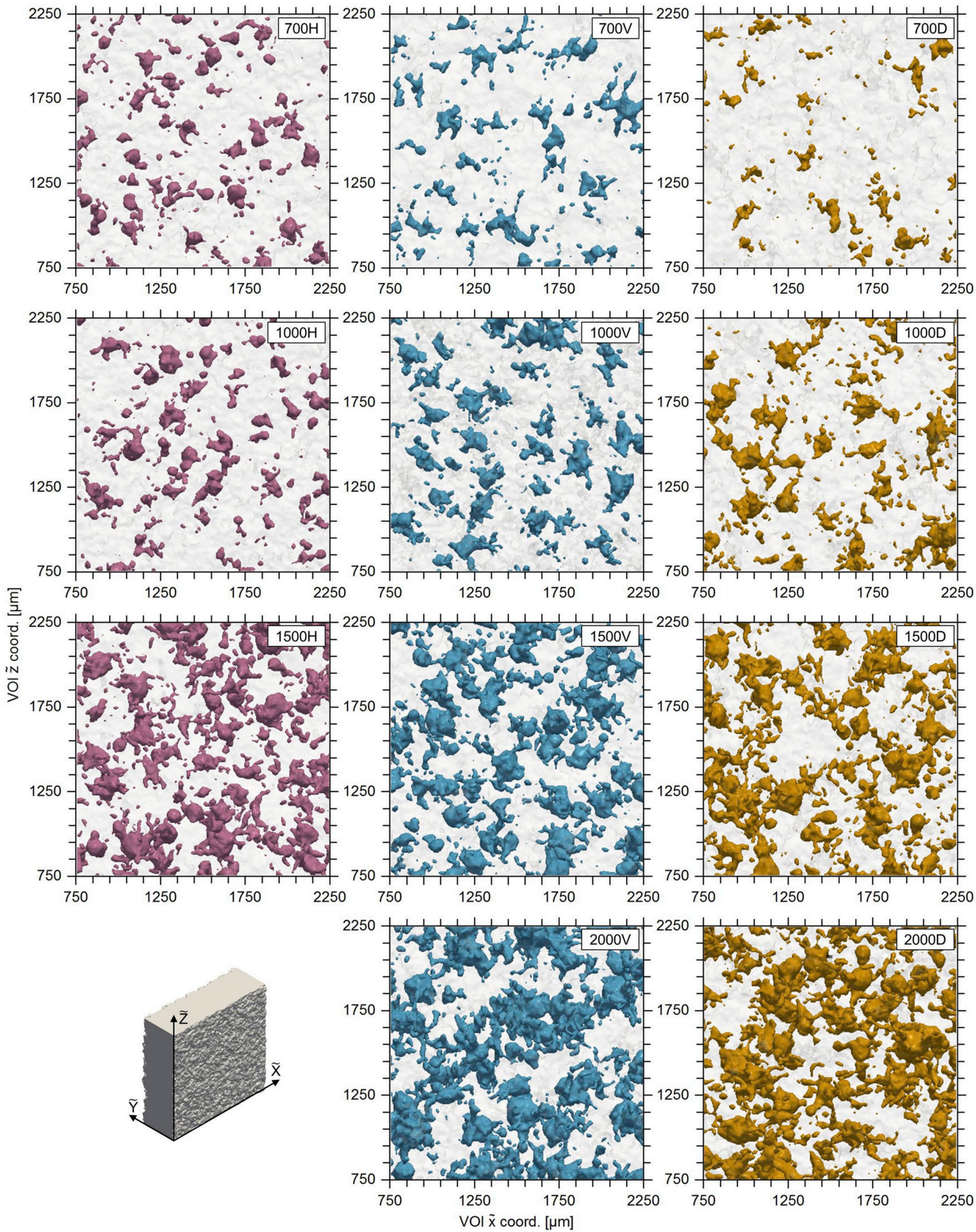

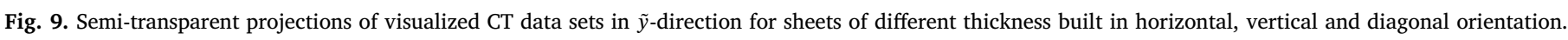


temperature levels during the building process. Considering the scanning patterns displayed in Fig. 2, one may presume that the absolute temperature level might increase with growing thickness of the sheets. In horizontal orientation, the thickness of the sheet is directly linked to the number of layers that are sintered subsequently. For vertically and diagonally built sheets, an increase in the thickness leads to a larger cross section area per layer comprising a higher amount of hatch scanning. From Fig. 8 it is apparent that horizontally built sheets overall present smaller pore sizes compared to thin sheets built in diagonal and vertical orientation. Fig. 6 reveals that sheet $700 \mathrm{H}$ and $700 \mathrm{~V}$ present quite similar overall porosity values, but significantly different pore number densities. Comparing the graphs referring to sheet $700 \mathrm{H}$ and $700 \mathrm{~V}$ in Fig. 8, a marked difference in pore size distribution can be recognized. Regarding sheet $700 \mathrm{~V}$, the major pore volume fraction can be found for equivalent pore diameters of $100 \mu \mathrm{m}-150 \mu \mathrm{m}$. By contrast, the total pore volume content of sheet $700 \mathrm{H}$ mainly refers to smaller pore sizes with an equivalent pore diameter $<100 \mu \mathrm{m}$. From Fig. 9, it can be observed that the medium and large pores detected within sheet $700 \mathrm{~V}$ again resemble interconnected smaller pores. By contrast, sheet $700 \mathrm{H}$ presents smaller pores with less complex shapes. Regarding the scanning patterns for vertically and diagonally built thin sheets in Fig. 2b, one could observe that the length of the hatch lines significantly differs in $\mathrm{x}$ - and $\mathrm{y}$-direction while the hatch lines in $\mathrm{x}$-direction are of minimal length depending on the thickness of the sheets. By contrast, the scanning patterns used to produce thin sheets in horizontal orientation include longer hatch lines with the same length in both directions corresponding to the diameter of the disc-shaped sheets (see Fig. 2a). It has been previously shown that decreasing the length of the scanning vectors causes a higher absolute temperature level of the polymer melt during the sintering process [23]. Thus, one may estimate that the absolute temperature levels during the building process of the thin sheets would be higher in vertical and diagonal orientation which could be a possible explanation for the more pronounced coalescence of pores compared to the thin sheets built in horizontal orientation $[20,22]$.

\subsubsection{Porosity distribution}

It has already been revealed in section 3.3.1 that all thin sheets possess a dense skin enclosing a more porous internal region. To further investigate the porosity distribution along the thickness of the thin sheets, Fig. 10 displays the calculated 2D porosity throughout the VOI in $\tilde{y}$-direction. Taking into account the orientation of the thin sheets within the building chamber (global coordinate system $\mathrm{x}, \mathrm{y}, \mathrm{z}$ ), this implies that the porosity distribution is evaluated along the printing direction (horizontal sheets: $700 \mathrm{H}-1500 \mathrm{H}$ ), along a direction parallel to the powder bed platform (vertical sheets: $700 \mathrm{~V}-2000 \mathrm{~V}$ ) and along a direction in a $45^{\circ}$ angle to the powder bed platform (diagonal sheets: 700D - 2000D).

From Fig. 10 it can be recognized that, apart from the dense skin, the local porosity inside the thin sheets mainly resemble the bulk value of residual porosity typically reported for LS parts (see section 3.3.1). This confirms the assumption that the lower overall porosity levels of the thin sheets primarily result from the large volume fraction of the dense skin compared to the total volume of the sample. Apart from that, the local porosity levels found in the internal regions of the sheets seem to slightly increase with growing thickness of the sheets, which becomes especially evident regarding the horizontally built sheets. This is consistent with findings reported by Tasch et al. [7] who used scanning electron microscopy to analyse the residual cross sections of thin-walled tensile test samples built in z-direction and observed an increased number of pores in the area of the hatch scanning with increasing wall thickness of the samples. The results suggest that, when comparing sheets of different thickness, occurring differences in the overall porosity could not only be attributed to the different ratio between the dense skin and the porous bulk region, but to different absolute porosity levels of the porous bulk as well.
Furthermore, Fig. 10 shows that all sheets built in horizontal orientation present regular porosity variations with a wavelength equal to the layer thickness of $100 \mu \mathrm{m}$ and the pores mainly concentrated between the layers. A similar oscillating behaviour of the porosity distribution along the printing direction was observed in previous research $[20,22]$, while it has been assumed that the porosity variations within a single layer result from the difficulty to achieve an efficient packing of the powder at the bottom of the layer. Regarding the porosity variations among the layers a possible explanation might be the powder feed system of the LS machine used to produce the thin sheets. This contains two feed chambers, where the powder is stored prior to being spread across the building platform by a radial moving blade, whereby fresh powder material is only delivered into the left feed chamber. Therefore, one may expect differences comparing the temperature of the powder layer supplied from the left and the right side, which may affect powder flowability and thus lead to different powder packing densities among the layers. Apart from that, the porosity variations among the layers could be due to the different directions of the laser scanning vectors resulting from the alternating $x-y$ scanning pattern (see Fig. 2) [15]. Looking at the porosity distribution of sheet $1500 \mathrm{H}$ it becomes evident that every second maximum is followed by a less distinct peak. This difference in the magnitude of two subsequent peaks indicates a trend towards a periodicity of porosity only every second layer. A similar porosity profile was observed by Pavan et al. [22] who investigated the porosity distribution within tensile bars produced with different process parameters and found a periodicity of the porosity every second layer when high energy densities where applied. Therefore, the different porosity profile observed for sheet $1500 \mathrm{H}$ may indicate a higher energy level compared to thinner sheets built in horizontal orientation which would be consistent with findings reported in section 3.3.2.

The porosity distribution along the thickness of vertically and diagonally built sheets differs significantly from the oscillating pattern found in horizontal orientation. All sheets present a significant increase of the porosity between the two dense skin regions. For sheet $2000 \mathrm{~V}$, one could observe two distinct maxima of porosity enclosing a more stable distribution of lower values in the internal region. The results are qualitatively similar to those obtained by Pavan et al. [14] who investigated the porosity distribution along the direction parallel to the powder bed platform inside a small cubic sample. A possible explanation for this effect might be found in the scanning strategy with the two outer maxima of porosity coinciding with the crossover from contour to hatch scanning. Supporting information is given by Dewulf et al. [20] who compared the porosity distribution of small cylinders built in vertical orientation using different scanning strategies. All patterns comprising both, hatch and contour scanning, caused the formation of a porous ring right inside the dense border of the cylinders coinciding with the contour lines. Regarding sheet $1500 \mathrm{~V}$ and 1000V, one could similarly suggest a trend towards a local minimum of porosity intercepting the inner porous region. Nevertheless, the described effect becomes barely noticeable with decreasing thickness of the sheets, which might be attributed to the decreasing amount of hatch scanning. As expected no similar behaviour could have been found for sheet $700 \mathrm{~V}$ which was produced applying a different scanning strategy without hatch scanning (see Fig. 2b). Regarding the scanning patterns displayed in Fig. 2, the porosity distribution of sheets built in diagonal orientation might be expected to resemble the one found in vertical orientation. However, the building process of the diagonally built sheets includes the sintered area to be shifted in $\mathrm{x}$-direction layer by layer which means that in z-direction the scanning pattern of one layer does not coincide with the one referring to the subsequent layer. This might have caused the porosity distribution presenting unordered fluctuations observed in diagonal orientation.

\subsubsection{Open porosity}

Since the determination of the total volume considers the outer topography of the thin sheets, the porosity reported up to here only 

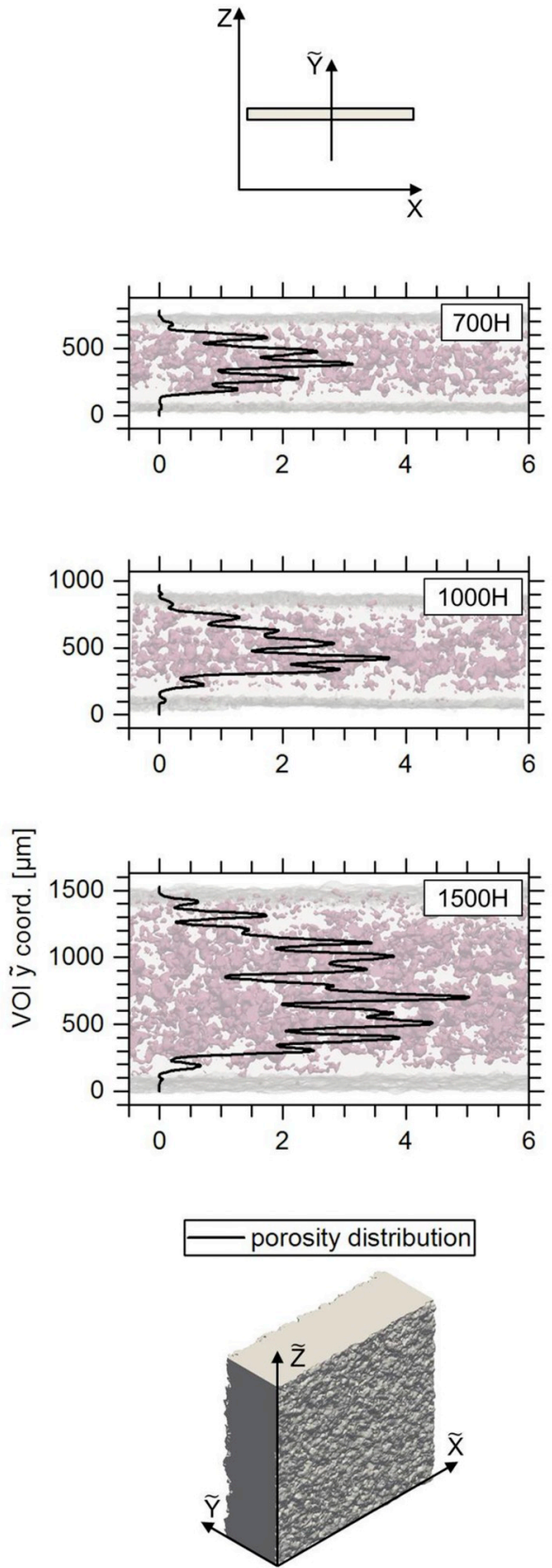
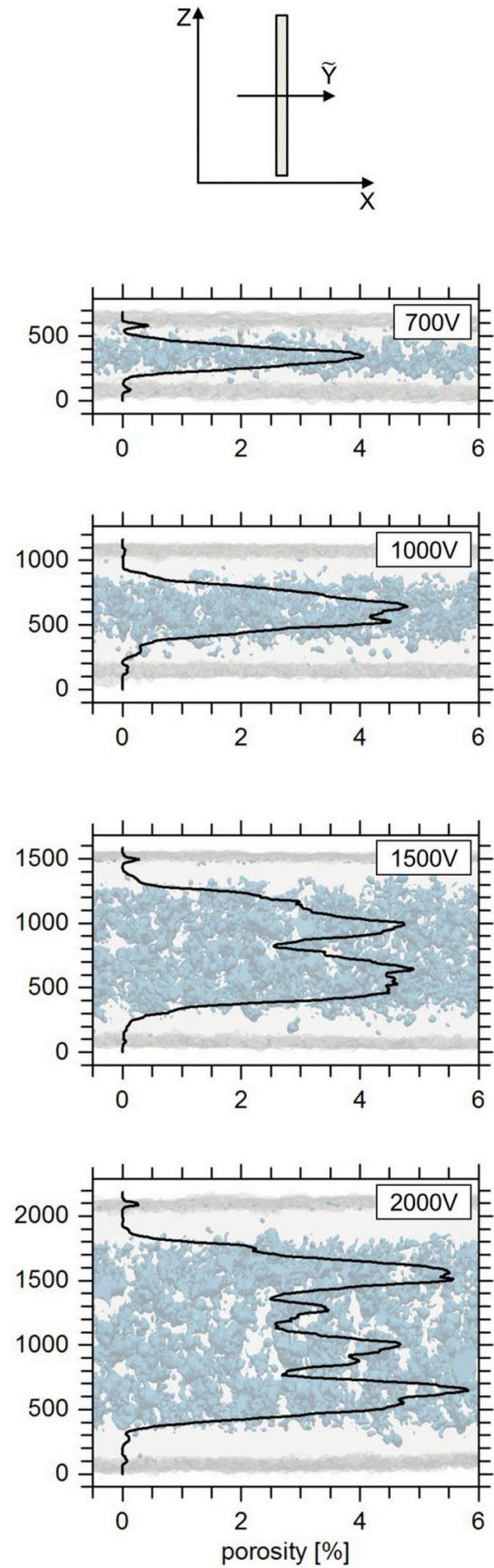
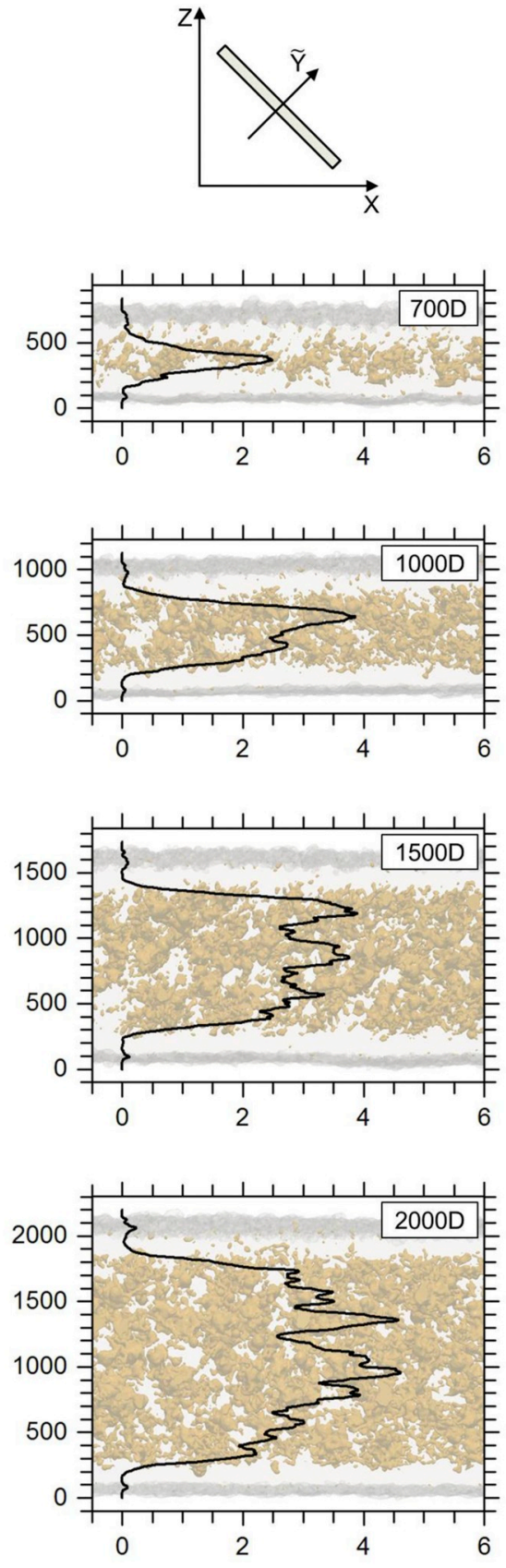

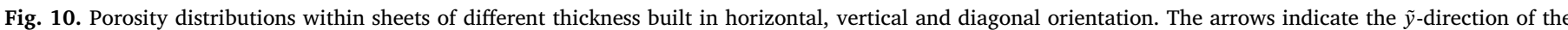
local VOI coordinate system relative to the global coordinate system referring to the building chamber.

included the internal pore volume fraction (closed porosity). This probably resulted in a lower prediction of porosity as pores which are connected to the surface of the sheets (open porosity) were not included. Fig. 11 again shows semi-transparent projections of the visualized CT data sets through $3 \mathrm{~mm}$ width in $\tilde{x}$-direction (local VOI coordinate system) for selected thin sheets. In order to clearly identify the open porosity, the total number of pore voxels (closed porosity) and matrix voxels has been merged to a single volume that represents the outer topography of the thin sheets.

In general, no marked open porosity can be observed. An exception is sheet $700 \mathrm{~V}$, which shows a single large, branched pore connected to the surface of the sheet. This suggests that the scanning strategy used to produce sheet $700 \mathrm{~V}$ might not have led to proper consolidation of the polymer material during the sintering process. An explanation could be the small number of scanning lines as well as the different exposure parameters referring to the edge line (see Fig. 2b).

Looking in detail at the images referring to sheet $700 \mathrm{H}$ and $1500 \mathrm{H}$, one recognizes small elongated open pores connected to the upwardfacing surface. This effect was similarly observed for all sheets built in horizontal orientation, indicating that trapped gases forming pores may show an upstream movement. Gogos et al. [24] described the migration of bubbles within a polymer melt by balancing the three forces acting on the bubble, that are buoyancy, weight and drag force. This showed that the velocity of a rising bubble mainly depends on the size of the bubble as well as the density and viscosity of the surrounding polymer melt. According to Wortberg et al. [25] the viscosity of the polymer melt is the predominant factor determining the coalescence of adjacent powder particles in LS processes. Wegner [23] revealed that a sufficient 

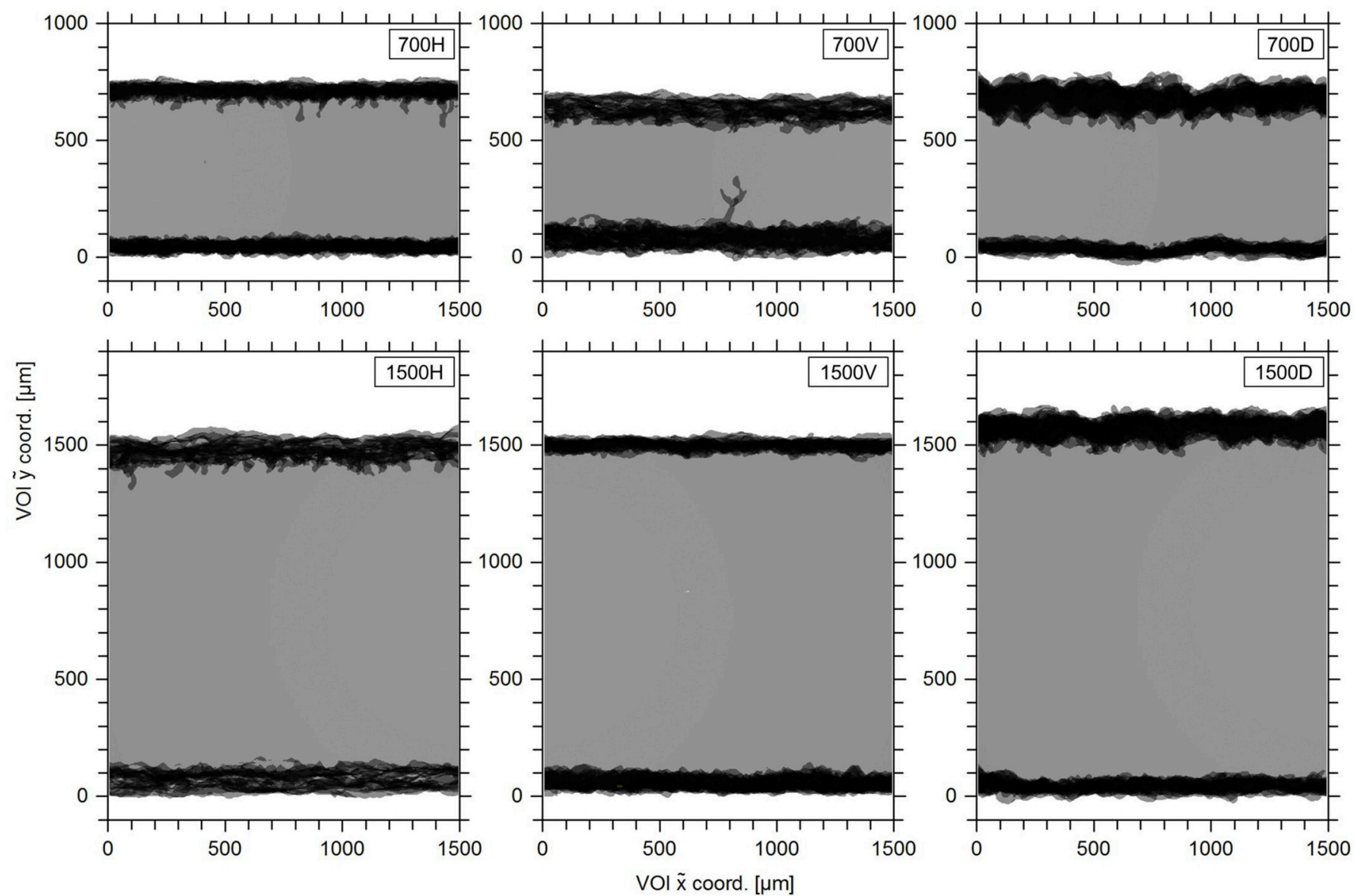

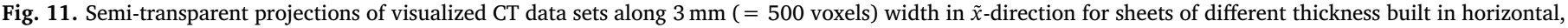
vertical and diagonal orientation. Pore and matrix voxels are merged to a single volume representing the outer topography of the thin sheets.

consolidation with a strong connection between single layers can be achieved when the polymer melt remains at a temperature level above the polymers melting temperature until new powder material is delivered across the sintered area. Thus, one could expect the polymer lasting in a dynamic state of low viscosity long enough to allow a migration of enclosed pores in z-direction. This hypothesis finds supporting information when looking at the images displayed in Fig. 7 which show the closed pores segmented from the polymeric matrix. Regarding horizontally built sheets, small pores as well as single larger pores obviously appear cumulated close to the upward-facing surfaces whereas only few closed pores can be found at the downward-facing side.

Regarding thin sheets built in diagonal orientation, one could expect elongated pores connected to the upward-facing surface for those regions which are not overlapped by the sintered cross section corresponding to the subsequent layer. This would further explain the low overall (closed) porosity levels observed for the diagonally built sheets as reported in section 3.3.1. Nevertheless, the expected effect could not have been clearly verified due to the marked surface roughness at the upward-facing side of the sheets which can be explained by the socalled stair-stepping effect [26].

\section{Conclusion}

This study analyzes the porosity characteristics of thin sheets produced by LS, thus contributing to a better understanding of LS processes. New insights into the pore formation within LS thin sheets could help to enhance ongoing work for improving LS processes in order to overcome existing restrictions regarding the production of thin-walled structures. The work shows experimental evidence that the porosity of
LS thin sheets strongly depends on the thickness and orientation within the building chamber. Different porosity characteristics might result from different scanning strategies and moreover, suggest different temperature levels during the building process of thin sheets with varying thickness produced in different build orientations. An important question for future studies is to determine how different scanning strategies and process parameters would influence the porosity of LS thin sheets. In any case, the results suggest that LS thin sheets will present different material properties, e.g. mechanical strength or permeability to gases and vapors, depending on their thickness and orientation within the building chamber. This should be taken into account when designing LS thin-walled hollow bodies for end-use applications. Future investigations will focus on the mechanical and physicochemical material properties of LS thin sheets depending on their thickness and build orientation.

\section{Data availability}

The raw/processed data required to reproduce these findings cannot be shared at this time as the data also forms part of an ongoing study.

\section{Acknowledgement}

The authors would like to gratefully thank Monika Gessler from EOS Electro Optical Systems GmbH (Krailling, Germany) for providing the samples for this research and for her valuable advice given during the investigations. Moreover, the authors acknowledge the financial support from the "Fritz-und-Brunhilde-Englisch-Stiftung" (Wiggensbach, Germany). 


\section{References}

[1] J.-P. Kruth, G. Levy, R. Schindel, T. Craeghs, E. Yasa, Consolidation of polymer powders by selective laser sintering, Proceedings of the 3rd International Conference on Polymers and Moulds Innovations, 2008, pp. 15-30.

[2] T. Laumer, K. Wudy, M. Drexler, P. Amend, S. Roth, D. Drummer, M. Schmidt, Fundamental investigation of laser beam melting of polymers for additive manufacture, J. Laser Appl. 26 (2014) 042003.

[3] J. Allison, C. Sharpe, C.C. Seepersad, A test part for evaluating the accuracy and resolution of a polymer powder bed fusion process, J. Mech. Des. 139 (2017) 100902-100902-100905.

[4] A. Wegner, G. Witt, Design rules for laser sintering, J. Plast. Technol. 8 (2012) 253-277.

[5] C.C. Seepersad, T. Govett, K. Kim, M. Lundin, D. Pinero, A designer's guide for dimensioning and tolerancing SLS parts, Solid Freeform Fabrication Symposium, Austin, TX, 2012, pp. 921-931.

[6] G.A. Adam, D. Zimmer, On design for additive manufacturing: evaluating geometrical limitations, Rapid Prototyp. J. 21 (2015) 662-670.

[7] D. Tasch, A. Mad, R. Stadlbauer, M. Schagerl, Thickness dependency of mechanical properties of laser-sintered polyamide lightweight structures, Addit. Manuf. 23 (2018) 25-33.

[8] H.C.H. Ho, I. Gibson, W.L. Cheung, Effects of energy density on morphology and properties of selective laser sintered polycarbonate, J. Mater. Process. Technol. 89 (1999) 204-210.

[9] D.L. Bourell, T.J. Watt, D.K. Leigh, B. Fulcher, Performance limitations in polymer laser sintering, Physics Procedia 56 (2014) 147-156.

[10] M. Pavan, T. Craeghs, J.-P. Kruth, W. Dewulf, Investigating the influence of X-ray CT parameters on porosity measurement of laser sintered PA12 parts using a designof-experiment approach, Polym. Test. 66 (2018) 203-212.

[11] S. Dupin, O. Lame, C. Barrès, J.-Y. Charmeau, Microstructural origin of physical and mechanical properties of polyamide 12 processed by laser sintering, Eur. Polym. J. 48 (2012) 1611-1621.

[12] D. Rouholamin, N. Hopkinson, An investigation on the suitability of micro-computed tomography as a non-destructive technique to assess the morphology of laser sintered nylon 12 parts, Proc. IME B J. Eng. Manufact. 228 (2014) 1529-1542.

[13] O. Ghita, E. James, R. Trimble, K.E. Evans, Physico-chemical behaviour of poly (ether ketone)(PEK) in high temperature laser sintering (HT-LS), J. Mater. Process. Technol. 214 (2014) 969-978.

[14] M. Pavan, T. Craeghs, J.-P. Kruth, W. Dewulf, Understanding the Laser Sintering of Polymers at Microscale Level by Using X-Ray Computed Tomography, Solid
Freeform Fabrication Symposium, Austin, TX, 2016, pp. 1112-1125.

[15] M. Pavan, M. Faes, D. Strobbe, B. Van Hooreweder, T. Craeghs, D. Moens, W. Dewulf, On the influence of inter-layer time and energy density on selected critical-to-quality properties of PA12 parts produced via laser sintering, Polym. Test. 61 (2017) 386-395.

[16] T. Stichel, T. Frick, T. Laumer, F. Tenner, T. Hausotte, M. Merklein, M. Schmidt, A round robin study for selective laser sintering of polyamide 12: microstructural origin of the mechanical properties, Optic Laser. Technol. 89 (2017) 31-40.

[17] T. Stichel, T. Frick, T. Laumer, F. Tenner, T. Hausotte, M. Merklein, M. Schmidt, A Round Robin study for selective laser sintering of polymers: back tracing of the pore morphology to the process parameters, J. Mater. Process. Technol. 252 (2018) 537-545.

[18] S. Rüsenberg, L. Schmidt, H. Schmid, Mechanical and physical properties-a way to assess quality of laser sintered parts, Proceedings of the 22nd International Solid Freeform Fabrication Symposium, 2011, pp. 239-251.

[19] M. Pavan, T. Craeghs, R. Verhelst, O. Ducatteeuw, J.-P. Kruth, W. Dewulf, CT-based quality control of laser sintering of polymers, Case Stud. Nondestruct. Test. Evaluat. 6 (2016) 62-68.

[20] W. Dewulf, M. Pavan, T. Craeghs, J.-P. Kruth, Using X-ray computed tomography to improve the porosity level of polyamide-12 laser sintered parts, CIRP Ann. - Manuf. Technol. 65 (2016) 205-208.

[21] N. Otsu, A threshold selection method from gray-level histograms, IEEE Trans. Sys., Man, Cybernet. 9 (1979) 62-66.

[22] M. Pavan, T. Craeghs, P.V. Puyvelde, J.-p. Kruth, W. Dewulf, Understanding the link between process parameters, microstructure and mechanical properties of laser sintered PA12 parts through X-ray computed tomography, Proceedings of the 2nd International Conference on Progress in Additive Manufacturing (Pro-AM 2016), 2016, pp. 569-574.

[23] A. Wegner, Theorie über die Fortführung von Aufschmelzvorgängen als Grundvoraussetzung für eine robuste Prozessführung beim Laser-Sintern von Thermoplasten (Diss.), Universitätsbibliothek Duisburg-Essen, 2015.

[24] G. Gogos, Bubble removal in rotational molding, Polym. Eng. Sci. 44 (2004) 388-394.

[25] J. Wortberg, G. Witt, C. Mielicki, A. Wegner, B. Gronhoff, Prediction of PA12 Melt Viscosity in Laser Sintering by a Time and Temperature Dependent Rheological Model, RT-Journal-Forum für Rapid Technologie, 2012.

[26] P. Delfs, M. Tows, H.-J. Schmid, Optimized build orientation of additive manufactured parts for improved surface quality and build time, Addit. Manuf. 12 (2016) 314-320. 